\title{
How to Put Usability into Focus: Using Focus Groups to Evaluate the Usability of Interactive Theorem Provers*
}

\author{
Bernhard Beckert \\ beckert@kit.edu
}

\author{
Sarah Grebing \\ sarah.grebing@kit.edu \\ Karlsruhe Institute of Technology (KIT)
}

\author{
Florian Böhl \\ boehl@kit.edu
}

\begin{abstract}
In recent years the effectiveness of interactive theorem provers has increased to an extent that the bottleneck in the interactive process shifted to efficiency: while in principle large and complex theorems are provable (effectiveness), it takes a lot of effort for the user interacting with the system (lack of efficiency). We conducted focus groups to evaluate the usability of Isabelle/HOL and the KeY system with two goals: (a) detect usability issues in the interaction between interactive theorem provers and their user, and (b) analyze how evaluation and survey methods commonly used in the area of human-computer interaction, such as focus groups and co-operative evaluation, are applicable to the specific field of interactive theorem proving (ITP).

In this paper, we report on our experience using the evaluation method focus groups and how we adapted this method to ITP. We describe our results and conclusions mainly on the "meta-level," i.e., we focus on the impact that specific characteristics of ITPs have on the setup and the results of focus groups. On the concrete level, we briefly summarise insights into the usability of the ITPs used in our case study.
\end{abstract}

\section{Introduction}

In recent years, the effectiveness of interactive theorem provers (ITPs) has increased to an extend that the bottleneck in the interactive process shifted to efficiency. While in principle large theorems are provable (effectiveness), it takes a lot of effort for the user interacting with the system (efficiency). This issue is recognized by the ITP-community and improvements are being developed. However, in contrast to properties like soundness or completeness, where rigorous methods are applied to provide evidence, the evidence for a better usability is lacking in many cases.

The work reported here is part of a project where we apply various survey and evaluation methods commonly used in the field of human-computer-interaction (HCI) to ITPs, including focus group discussions, usability testing, and user experience questionnaires. Since expertise from both fields (ITP and $\mathrm{HCI}$ ) is required, we cooperate with user experience experts from DATEV eG within the BMBF-funded Software Campus programme (they are well-versed in the ergonomic evaluation of standard software).

Our contribution in this paper is a description of how to use focus group discussions, or focus groups for short, to evaluate the usability of ITPs. Moreover, we explain what kind of questions one can expect to answer using this focus groups, which are structured group discussions guided by a moderator.

We report on two experiments we have conducted, applying the focus group method to two different ITPs: the tactical theorem prover Isabelle/HOL [16] and the interactive program verification system $\mathrm{KeY}[5]$.

\footnotetext{
${ }^{*}$ The work presented here is part of the project Usability of Software Verification Systems within the BMBF-funded programme Software Campus.

C. Benzmüller and B. Woltzenlogel Paleo (Eds.):

Eleventh Workshop on User Interfaces for Theorem Provers (UITP 2014)

EPTCS 167, 2014, pp. 4-13 doi 10.4204/EPTCS.167.3
} 
We describe our setup for the focus group discussions, what needs to be done in preparation for the discussions, and how the discussions are evaluated to draw conclusions in the post-processing phase. We hope that our experiences help others to conduct focus groups in this field to improve the quality of ITP user interfaces.

\section{Related work.}

As mentioned above, the ITP community has noticed the need to evaluate and improve usability, but so far structured usability evaluation methods have rarely been applied to ITPs. Related work where this has been done, includes the following: In previous work [4], we have performed a questionnaire-based evaluation of the KeY system with a questionnaire based on Green and Petre's cognitive dimensions questionnaire [6] in order to get a first impression of the user's perception and to develop first hypotheses about the usability of the KeY system. Kadoda et al. evaluated proof systems using Green and Petre's Cognitive Dimensions questionnaire to develop a list of desirable features for educational theorem provers [11]. Aitken and Melham evaluated the interactive proof systems Isabelle and HOL using recordings of user interactions with the systems in collaboration with HCI experts. During the proof process the users were asked to think-aloud and after the recordings the users were interviewed. Their goal was to study the activities performed by users of interactive provers during the proof process to obtain an interaction model of the users. As usability metric they propose to use typical user errors and they compared provers w.r.t. these errors [2, 3, 1]. Based on the evaluation results also suggestions for improvements of the systems have been proposed by the authors including, besides others, improved search mechanisms and improved access to certain proof relevant components. Jackson et al. used co-operative evaluation methods on the CLAM Proof Planner. Users were asked to perform predefined tasks while using the "think aloud technique" to comment on what they where doing [10]. Questionnaires and interviews were used by Vujosevic and Eleftherakis to answer the question why Formal Methods Tools are not used in industry [18]. In the context of this work, also usability aspects of several formal methods tools, such as the Alloy Analyzer, were evaluated. For improving the interface of the prover NuPRL, a self-designed questionnaire was used to evaluate the users' perceptions of the interface [8].

Structure of this paper. We give an introduction to the focus group method in Section 2 and describe our experiments using focus groups to evaluate the usability of ITPs. The script used to guide the discussions is discussed in Section 3. In Section 4, we present the results of the experiments. Here, we focus on the meta-level, i.e., on the impact that specific characteristics of ITPs have on the setup and the results of focus groups. On the concrete level, we briefly summarise insights into the usability of the ITPs used in our case study. Finally, in Section 5 , we discuss future work.

\section{Survey Method and Adaptation to ITPs}

Focus Groups. A focus group is a discussion of five to ten participants guided by a moderator. The moderator uses a prepared script to initiate and structure the discussion which typically lasts about one to two hours. Focus groups are a standard method in many areas to explore opinions about specific products or topics, e.g., in market research. In the field of human-computer interaction, they are used to explore user perspectives on software systems and their usability.

The Challenge of Conducting a Focus Group. Focus groups require less participants than evaluations using questionnaires and the effort for conducting the discussion is less than that of one-on-one interviews [17, 9]. Still, it is a non-trivial task to conduct a focus group. The discussions have to be well-structured as well as lively and open to be productive. And it is a challenge to steer the discussions towards the topics of interest without predisposing possible answers or biasing the results in other ways. 
Fortunately, we can draw from a big body of knowledge on how to conduct focus group discussions (e.g., [15, 7]).

Focus Groups for ITPs. We have conducted two focus group discussions on the usability of ITPs, one for the KeY system [5] and one for Isabelle/HOL [16]. The setup and topics of both discussions were the same to make the results easier to compare. In the remainder of this section we describe our course of actions specifically for ITPs. We discuss the three phases of an evaluation using focus groups, namely pre-processing, the discussion itself, and the post-processing.

\subsection{Pre-Processing}

Selecting the Participants. In general, the composition of the focus group should be representative for the user base of the tool being evaluated. But participants may also be selected from certain sub-groups, such as beginners or experts. Both the level of expertise in the relevant domain and the experience level for the evaluated tool are relevant criteria. It is also crucial to have a group of participants who are motivated and keen to debate.

Our Setup: The participants for our experiments were recruited using personal contacts to the relevant communities. We ensured that each group included novice, intermediate, and expert users in different proportions. Besides that, the only criterion for selection was that participants had to be open about the idea of focus group discussions (mostly they were interested in a new experience and to learn something new about using their tools). Most participants were Master or PhD students, who had used KeY resp. Isabelle for their thesis work. We reimbursed participants' travel expenses but they were not paid a fee. The KeY group had seven and the Isabelle group five participants. In the Isabelle group we had one novice, two intermediate and two experts users. In the KeY group we had one novice, two intermediate and four expert users.

The Moderator. The moderator must not be one of the stake holders and must be neutral in his or her opinion about the evaluated software. This excludes, for example, developers of the evaluated tool. Nevertheless, the moderator must understand the issues that are discussed. A well-prepared and experienced moderator can greatly improve the results of a focus group discussion.

Our Setup: We had two different moderators, one for each discussion. Both were computer scientists working in academia but not in the area of ITP. As they were not expert moderators, they got an extensive training and briefing prior to the discussions.

The Technical Setup. It is advisable to use two adjacent rooms, one for the discussion itself and one for observers, including the experimenters and some domain experts (e.g., developers of the ITP). The discussion is recorded with at least one camera and several microphones and is transmitted live to the observation room. This setup has to be well tested beforehand as any technical problem can seriously effect the post-processing of the recorded discussion. It is useful to provide a feedback channel from the observation room to the moderator (using a headset) to give hints and provide relevant domain knowledge.

Our Setup: We followed the advices about the spatial setup and used two adjacent rooms (one for the discussion, one for the observers) with a glass window between them. The technical equipment consisted of one camera and four microphones for recording, a back channel from the observers to the moderator's headset, and lecture recording software capable of recording and live streaming.

The Script. The script contains all tasks and all questions for the focus group. Only neutral questions can be asked explicitly (e.g., "Please name one good and one bad feature of the tool."). Non-neutral questions such as "Is feature $X$ useful?" are included in the script but are not asked explicitly. Instead it is the moderator's task to guide the discussion into the direction of these questions, e.g., by digging 
deeper when a participant brings up a certain issue. Of course, the moderator has to carefully balance neutrality and the desire to steer the discussion in a certain direction. The topics in the script should build on each other in a meaningful way, e.g., from a general topic to a specific topic [7].

Our Setup: The questions of the scripts for our conducted focus groups are described in Sec. 3 . The planned duration for both groups was 2 hours. Due to lively discussions, the actual duration was 2.5 resp. 3 hours.

\subsection{The Discussion}

The discussion itself starts with a round-robin introduction of the participants and some small warm-up tasks, and it ends with a cool-down task that allows to summarize the content of the discussion. The main part consists of sub-discussions that are related to specific topics such as usability aspects, tool features, etc. Each topic is introduced by the moderator, possibly using example problems, mock-ups of new features or similar material. After the recorded part of the discussion ends, there should be time for questions and feedback from the participants and the moderator (even if that part is not recorded, it is useful to take notes).

Our Setup: Our discussion had three stages: the warm-up stage, the main stage and the cool-down stage.

The discussion was carried out according to the script, which is explained in detail in Sec.3. All in all both discussion groups were lively and the participants engaged well in the discussion. Our impression was that the participants were open towards this method and were upfront about their systems. Our moderators were not experienced with moderations tasks and sometimes asked suggestive questions like "Do you all share person A's opinion?". While this required an extra-careful analysis of the transcribed material, the damaging effect of such questions for the results was minimal. A thorough analysis of the video material showed that often the group or certain participants confirmed or denied a statement before the corresponding suggestive question occurred.

After the discussion, all participants had the chance to have a small offline talk with all project members and ask questions as well as express opinions about the focus group without being recorded. We believe these offline discussions were a good opportunity for the participants to gain more information about the focus group method as well as for the clarification of some issues which may not have been addressed sufficiently in the discussion. Therefore, we suggest to take notes in this offline part.

\subsection{Post-processing}

In the post-processing phase the recorded material has to be transcribed, analyzed, and evaluated.

The first analysis step is to check if the participants conformed to the expected user types or whether the group has to be divided into sub-groups (e.g., beginners and experienced users). Given this grouping, opinions expressed within the focus group can be associated with their user type during analysis, if applicable. This association allows to draw first conclusions for each user type.

One method suitable for categorizing and extracting the information from the discussion is qualitative content analysis [13]. Similarly to the classification of users, the material has to be categorized and opinions have to be assigned to the categories. The categories are based on the research question, the questions asked during the discussion, as well as the opinions given by the participants [14].

First, for each explicit and implicit question in the script, an own top-level category is defined, e.g., "Strengths of the system related to the proof process". Then the discussion is analyzed and for each opinion related to the top-level, if a suitable subcategory already exists, the opinion is assigned to that 
subcategory. If not then a new subcategory is introduced and the opinion is assigned to this category. For example, assume that the subcategory "user interface" was already defined and an opinion of one of the participants is: "The user interface is great!". Then this opinion would be assigned to the subcategory "user interface".

During this analysis, it is important to remain objective, to take all stated opinions into consideration, and to avoid bias when interpreting what has been said. It is useful to involve several persons in this task, including the moderator. When the material is categorized a revision of the categories may be done. For example some categories may be merged together to a larger or more abstract category. After the categorization the opinions assigned to each of the subcategories have to be carefully analyzed and conclusions for the usability of this subcategory have to be drawn. This is a creative process and depends on the experience of the project members as well as the underlying tasks and research questions. Nevertheless, we give some suggestions, where to draw attention to during analysis. It may be advisable to also take care which user type stated the opinion, as beginners often have different usability issues than intermediate or expert users. Also the reactions of the group should be taken into account, because an issue which the majority of the group agrees on might be an issue which the majority of the users in general might have as well. Attention should also be drawn to issues occurring with a higher frequency than others, regardless of the part or phase of the discussion they are expressed. There might be a correlation between the frequency and the relevance of an issue.

\section{The Script}

The main questions and tasks in the script were the same for both discussions as we wanted to compare the results. The only differences were adaptations of the questions and tasks to the different terminology and adaptations of feature mock-ups to the specifics of the two systems. The full scripts for our experiments are available at http://formal.iti.kit.edu/grebing/SWC (as the discussions were conducted in German, the original scripts are in German, but a translation to English is provided as well). Table 1 gives a summary of the explicit questions for the participants. Our discussion was divided into three parts: the warm-up stage, the main stage and the cool-down stage. All three parts will be described in detail in the following.

\subsection{Warm-Up Tasks}

As warm-up task, we asked about typical application areas of the systems and about their strengths and weaknesses related to the proof process. Our intention for this part of the discussion was twofold. Firstly, we wanted the participants to slowly focus on the proof process of their system and "warm up" for the main part of the discussion. Secondly, our goal was to retrieve the advantages and disadvantages of the systems to draw conclusions about desirable features for interactive theorem provers. In addition, we expected to retrieve detailed information about the systems such that we can name the issues and give advices how to improve the systems.

\subsection{Main Part}

The main part of the discussion covered two topics:

1. The proof process: What does the proof process look like? How does the tool give support to the user during the process? 
1. Name typical use cases of the system.

2. Name a strength of the system related to the proof process.

3. Name a weakness of the system related to the proof process.

For the global and the local proof process:

1. How do you conceive a formalization/specification for a given problem?

(a) Please try to sketch the process.

(b) Please point out steps of the process during which you get help/feedback from the system (if any).

(c) Do you repeat certain sequences of steps during the process? If so, please mark these loops.

2. (Discussion)

(a) How do you rate the feedback you get from the system? (If negative: Where would be room for improvements?)

(b) Which steps of the process consume most of your time? Why?

(c) Which steps of the process annoy you? Could they be automated?

(d) What do you do if you get stuck?

(e) How do you rate the granularity of the proofs (in the local process)?

For the mechanisms:

1. Please describe the presented mechanism.

2. Please rate the presented mechanism.

3. What do you make of the approach?

1. Be creative and describe your ideal interactive proof system. Disregard technical restrictions apart from the effectiveness of the automated proof search. Name capabilities the system should definitely have. Name properties it must not have.

Table 1: Summary of the questions from the script for the focus groups. 
2. Mechanisms for understanding proof states: We confronted the participants with mechanisms that might help them to understand the current state of a proof during the proof process.

Topic 1: The Proof Process. We divided the discussion for this topic into two parts, namely the global proof process (finding the right formalization and decomposing the proof task) and the local proof process (proving a single lemma or theorem). For each part, participants were asked to describe their typical proof process and discuss where the prover gives support and where support is missing. We also asked what the most time-consuming actions are.

By discussing the proof process, participants remember their typical interactions with the system in the past. This supports the subsequent discussion of how users get assistance from the systems during the proof process. Based on the participants' retrospection we hope to identify repetitive tasks or time consuming tasks, and parts where system feedback is missing.

Also, we expect information on participants' use of the systems to solve particular tasks, on actions/phases or where they switch to other tools (e.g., text editors or pen and paper), on how they inspect the proof state, and on how they guide the prover in finding a proof. We also expect ideas from the participants on how and where they would improve the systems.

Topic 2: Mechanisms for Understanding Proof States. For the second topic, we did not just ask for available or missing mechanisms. Instead we initiated a more focussed discussion by presenting mock-ups of mechanisms not yet built into the tools. The mock-ups were presented as a sequence of UI screenshots that have been modified according to the effect of a mechanism. These sequences of screenshots showed how to invoke the mechanisms and the corresponding effect of the mechanism 1 . The purpose of the presented mechanisms was to support users in understanding proof situations. The design of the particular mechanisms was based on first hypotheses. First informal questionings of some users influenced the design as well.

The mock-ups included (a) a mechanism for tracing formulas, terms, and variables that are generated during proof construction back to the original proof goal (for both tools), (b) a visual support for proof management that shows which lemmas contribute to a proof (for Isabelle), and (c) a mechanism for highlighting local changes between two adjacent nodes in the proof tree (for KeY). Thus, we made use of the possibility to use focus groups to get a first assessment of new features.

For all presented mechanisms we had the same course of action and questions. First, the participants were asked to describe what they believe the mechanism does (i.e., the mechanism was not explained by the moderator). This was done both to avoid bias introduced by the moderator and to see if the mechanism is intuitive. Then, the participants were asked for their opinion on the usefulness of the mechanism. We expected to gain feedback about the presentation of the mechanism. If the participants had needed too much time to understand the functionality, we would have had to revise the presentation of the mechanism. We also had to some extent the opportunity to gain feedback for different presentations of the same functionality, such as the mechanism as own functionality with an own window or incorporated in the provers graphical user interface.

With these mechanisms as starting point, we expected to get a discussion going about the usability problems w.r.t. the proof process where the presented mechanisms might help, and to gain detailed insight into what annoys the users and in which way they would like to see their system improve. Additionally, we expected answers to the question of which mechanism should be implemented first.

\footnotetext{
${ }^{1}$ The screenshots are available at http://formal.iti.kit.edu/ grebing/SWC/
} 
Table 2: Time and effort needed for conducting the two experiments (approximate).

\begin{tabular}{lrllrl}
\hline script preparation & $20-40$ & hours & discussions & 5.5 & hours \\
recruiting participants & 10 & hours & transcription of recording & 20 & hours \\
briefing moderators & 40 & hours & analysis & $80-160$ & hours \\
technical setup and testing & 8 & hours & & & \\
\hline
\end{tabular}

\subsection{Cool-Down Task}

For the cool-down task, we asked the participants to be creative and imagine their ideal interactive proof system.

The main idea behind the cool-down task is that the participants leave the discussion with a positive experience. Our intention was that we also gain some more, possibly creative, ideas on what features an ideal verification system should or should not incorporate.

\section{Results and Conclusion}

\subsection{Meta-level: Using Focus Groups to Evaluate ITPs}

Our experiments clearly show that the focus-group method is not just for business software but can be applied successfully to specialized tools such as ITPs. We gained lots of insight from our experiments.

Focus groups are well suited for an explorative and qualitative investigation of strengths and weaknesses in usability and the usefulness of new features. They are particularly useful for systems with a relatively small user base such as ITPs. Focus groups are a huge step towards objective and reproducible experiments in the area of usability, even though they do not provide precise quantitative data. Another strength of focus groups is that participants voice ideas and discuss issues that they would not have talked about in single interviews, when topics are brought up by other participants. Our experience is that we gain detailed feedback to usability issues of the system. In addition, the discussions provide an understanding of how users use the system to achieve their goals.

The effectiveness of focus groups and their advantages over unstructured discussions, however, do not come for free. Conducting focus group discussions takes careful preparation and is a non-trivial task. The effort and the required time is considerable. The approximate work-load of our experiments is given in Table 2 (person-hours for both experiments, not including the participants' and moderators' effort).

Our experiments support our hypothesis that the focus groups can also be performed successfully when computer scientists are employed as moderators instead of hiring professional moderators. Their inexperience in moderating sometimes lead to biased questions and also contributed to the discussions running over time. But their familiarity with the basic terms of logic and theoretical computer science allowed the discussions to proceed smoothly without spending too much time on clarifying basic notions during the discussion.

We believe that it is possible to repeat the focus group discussions also for other theorem provers with the necessary preparation. The parties involved in preparing and conducting a focus group need some knowledge about the system under evaluation and should have some ideas where issues arise during the proof process. Also ideas for improvement are specific to each system and therefore the involved parties should have worked out some ideas such that it is possible to provide mock-ups for the discussion. The experiments we have performed here can be repeated by using this description of our setup and the scripts available. 


\subsection{The Kind of Insights to be Gained on the Concrete Level}

We identified strengths and weaknesses of the two systems, which mostly can be generalized to most or all ITPs. A typical weakness is, for example, an inadequate understanding of what the effect of automatic proof search strategies is. Users may lose the comprehension of the proof by applying automatic strategies, as in some cases the strategies do not give feedback which rules or transformations they apply and leave the user with a proof state that differs from the last seen state.

Also, technical issues that are annoying for the user and compromise efficiency, were mentioned, e.g., unstable proof loading mechanisms or a user interface that is not sufficiently reactive. These answers point to where the systems' usability could be improved in particular.

Discussions about the proof process gave us insights into the feedback mechanisms of both systems that support the proof process, e.g., the different automatic tools in Isabelle. Also issues that arise during the processes have been mentioned by the participants and ideas for improving certain aspects. These include for example presentations of the proof tree. We learned how the users use their systems to accomplish certain proof tasks and where they switch to other systems in order to get a better understanding of the current proof state, for example by using an external text editor. By showing mock-ups of improvements we gained lively feedback and opinions about the presented mechanisms. With these opinions it should be possible for us to improve our mechanisms and prototypically implement them. From the opinions we try to draw conclusions about which mechanisms are more desired than others and therefore get hints what to develop first.

The creative task at the end of the discussion lead to interesting and creative interaction mechanisms for ITPs, but also general desirable features for ITPs have been mentioned. Some of these features are already part of the systems; others need improvement.

\section{Summary and Future Work}

To sum up, we have presented a method to qualitatively evaluate the usability of systems with a rather small user base. We have also shown, that it is possible to perform this evaluation without expert moderators, when being aware of this fact during the analysis of the transcribed material. We made the experience that we gain insight onto the usability of ITPs using this method and that we can formalize first hypotheses about usability issues of the systems as well as a first lits of desirable features for ITPs.

A full analysis and interpretation of the recorded and transcribed material is currently being done. This will result in a detailed report on desirable features for interactive theorem provers.

The mechanisms that attracted interest during the discussions need to be further developed and prototypically implemented. To ensure that the mechanisms suits the users needs and to evaluate whether they increase the usability, we will use usability testing. In addition, we will apply the User Experience Questionnaire method [12] to assess the usability of the KeY system quantitatively. In this evaluation, we will determine, whether such general-purpose questionnaires are helpful in evaluating the usability of ITP systems, or whether more adaptable solutions are needed.

\section{Acknowledgements}

We thank the participants in our focus group discussions on the usability of $\mathrm{KeY}$ and of Isabelle and, in particular, the two moderators for their great work. In addition, we thank our project partners from DATEV eG for sharing their expertise in how to prepare and analyze focus group discussions. Florian Böhl was supported by MWK grant "MoSeS". 


\section{References}

[1] J. S. Aitken, P. Gray, T. Melham \& M. Thomas (1998): Interactive Theorem Proving: An Empirical Study of User Activity. Journal of Symbolic Computation 25(2), pp. 263-284, doi 10.1006/jsco.1997.0175.

[2] J. Stuart Aitken \& Thomas F. Melham (2000): An analysis of errors in interactive proof attempts. Interacting with Computers 12(6), pp. 565-586, doi 10.1016/S0953-5438(99)00023-5

[3] Stuart Aitken, Philip Gray, Tom Melham \& Muffy Thomas (1995): A Study Of User Activity In Interactive Theorem Proving. In Chris Johnson, editor: Task Centred Approaches To Interface Design: Glasgow Interactive Systems Group Research Review, Department of Computing Science, University of Glasgow, pp. 195-218. GIST Technical Report G95.2.

[4] Bernhard Beckert \& Sarah Grebing (2012): Evaluating the Usability of Interactive Verification System. In: Proceedings, 1st International Workshop on Comparative Empirical Evaluation of Reasoning Systems (COMPARE), Manchester, UK, June 30, 2012, CEUR Workshop Proceedings 873, CEUR-WS.org, pp. $3-$ 17. Available at http://ceur-ws.org/Vol-873

[5] Bernhard Beckert, Reiner Hähnle \& Peter H. Schmitt, editors (2007): Verification of Object-Oriented Software: The KeY Approach. LNCS 4334, Springer-Verlag, doi:10.1007/978-3-540-69061-0.

[6] Alan Blackwell \& Thomas R. Green (2007): A Cognitive Dimensions Questionnaire (Version 5.1.1). www.cl.cam.ac.uk/ afb21/CognitiveDimensions/CDquestionnaire.pdf.

[7] Stanley Caplan (1990): Using focus group methodology for ergonomic design. Ergonomics 33(5), pp. 527-533, doi:10.1080/00140139008927160 Available at http://www.tandfonline.com/doi/abs/10. $1080 / 00140139008927160$.

[8] James Cheney (2001): Project Report - Theorem Prover Usability. Technical Report. Report of project COMM 641, available at/http://homepages.inf.ed.ac.uk/jcheney/projects/tpusability.ps

[9] Janice Elliott, Sara Heesterbeek, Carolyn J. Lukensmeyer \& Nikki Slocum (2005): Participatory Methods Toolkit: A practitioner's manual. Technical Report, King Baudoin Foundation and Flemish Institute for Science and Technology Assessment (viWTA).

[10] Michael Jackson, Andrew Ireland \& G. Reid (1999): Interactive Proof Critics. Formal Aspects of Computing 11(3), pp. 302-325, doi $10.1007 / \mathrm{s} 001650050052$.

[11] Gada Kadoda, Roger Stone \& Dan Diaper (1996): Desirable Features of Educational Theorem Provers: A Cognitive Dimensions Viewpoint. In: Proceedings of the 11th Annual Workshop of the Psychology of Programming Interest Group.

[12] Bettina Laugwitz, Theo Held \& Martin Schrepp (2008): Construction and Evaluation of a User Experience Questionnaire. In Andreas Holzinger, editor: HCI and Usability for Education and Work, Lecture Notes in Computer Science 5298, Springer Berlin Heidelberg, pp. 63-76, doi:10.1007/978-3-540-89350-9_6.

[13] Philipp Mayring (1996): Einführung in die qualitative Sozialforschung - Eine Anleitung zu qualitativem Denken (Introduction to qualitative social research). Weinheim: Psychologie Verlags Union.

[14] Philipp Mayring (June 2000): Qualitative Content Analysis. Forum: Qualitative Social Research 1(2). Online Journal, 1(2). Available at: http://www.qualitative-research.net/index.php/fqs/article/view/1089 [Date of access: 08, 2014].

[15] Jakob Nielsen (1993): Usability Engineering. Morgan Kaufmann Publishers Inc., San Francisco, CA, USA.

[16] Tobias Nipkow, Lawrence C. Paulson \& Markus Wenzel (2002): Isabelle/HOL: A Proof Assistant for HigherOrder Logic. LNCS 2283, Springer, doi:10.1007/3-540-45949-9.

[17] Petra Vogt \& Sven Heinsen (2003): Usability praktisch umsetzen: Handbuch für Software, Web, Mobile Devices und andere interaktive Produkte. Hanser.

[18] V. Vujosevic \& G. Eleftherakis (2006): Improving Formal Methods' Tools Usability. In G. Eleftherakis, editor: 2nd South-East European Workshop on Formal Methods (SEEFM 05), Formal Methods: Challenges in the Business World, Ohrid, 18-19 Nov 2005, South-East European Research Centre (SEERC). 